\title{
Rescaling of diffusion coefficients in two-time scale chemical systems
}

Damián E. Strier, and Silvina Ponce Dawson

Citation: The Journal of Chemical Physics 112, 825 (2000); doi: 10.1063/1.480650

View online: https://doi.org/10.1063/1.480650

View Table of Contents: http://aip.scitation.org/toc/jcp/112/2

Published by the American Institute of Physics

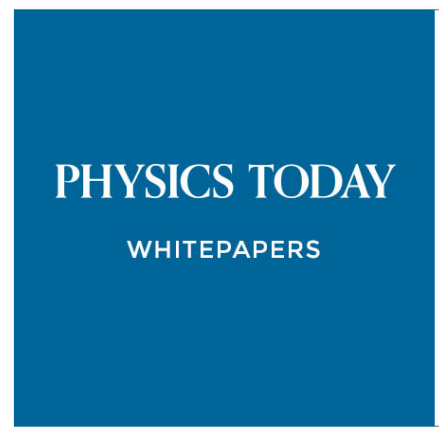
systems can do 


\title{
Rescaling of diffusion coefficients in two-time scale chemical systems
}

\author{
Damián E. Strier ${ }^{\mathrm{a})}$ and Silvina Ponce Dawson ${ }^{\mathrm{b}}$ \\ Departamento de Física, Facultad de Ciencias Exactas y Naturales, U.B.A., Ciudad Universitaria, \\ Pabellón I, (1428) Buenos Aires, Argentina
}

(Received 14 June 1999; accepted 1 October 1999)

\begin{abstract}
We study reaction-diffusion systems which involve processes that occur on different time scales. In particular, we apply a multiscale analysis to obtain a reduced description of the slow dynamics. Under certain assumptions this reduction yields a new set of reaction-diffusion equations with rescaled diffusion coefficients. We analyze the Selkov model [E. E. Selkov, Eur. J. Biochem. 4, 79 (1968)] and the ferrocyanide-iodide-sulfite reaction [E. C. Edblom et al., J. Am. Chem. Soc. 108, 2826 (1986)] to determine whether the rescaling in this case may account for the difference of diffusivities that the formation of certain types of patterns requires. (C) 2000 American Institute of Physics. [S0021-9606(99)51848-5]
\end{abstract}

\section{INTRODUCTION}

Self-organization in far-from-equilibrium systems has become a major topic of scientific research during the last decades. ${ }^{1}$ In particular, pattern formation in chemical systems has attracted a great deal of attention due to its possible applications to biological systems. ${ }^{2}$ One of the first clear steps to generate such interest was the work of Turing in $1952,{ }^{3}$ who pointed out the possibility of finding steady nonhomogeneous structures as the result of the interplay between nonlinear reaction kinetics and diffusion processes. Turing's work enhanced the study of reaction-diffusion systems, both from a theoretical and an experimental point of view. In fact, reaction-diffusion processes have become a key problem to push forward our knowledge about the mechanisms of pattern formation. Reaction-diffusion equations have been successfully applied to model different phenomena in fluid dynamics, chemical reactions, and dendritic growth, among others. Turing's main concern was the far reaching implications of this mechanism for generating patterns in biological systems (see, e.g., Ref. 2). Although there is no definite proof that this type of mechanism is at work in any real biological system, there are some very promising recent results. ${ }^{4}$

Turing patterns were not observed in laboratory experiments until 38 years after their theoretical description. ${ }^{5}$ This was due in part to the fact that Turing patterns need the chemicals to diffuse at different rates, and this was hard to achieve in the dilute aqueous systems that the community was focusing on. The experiments were done using the chlorite-iodide-malonic acid (CIMA) reaction. ${ }^{5}$ The analyses of these experiments ${ }^{6,7}$ showed that the interaction between the reacting and diffusing species with other immobile chemical complexes present in the system has a paramount importance for the patterns to occur. Lengyel and Epstein ${ }^{6}$ proposed a kinetic mechanism for the CIMA reaction and argued qualitatively that the gel where the reaction proceeds (loaded with starch molecules that also "trap" iodide ions)

\footnotetext{
a)Electronic mail: strier@df.uba.ar

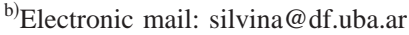

effectively reduces the diffusion coefficient of iodide. In this way, the activator and inhibitor species diffuse at different rates, as required for Turing patterns to exist. The more rigorous linear analysis of Ref. 7 was also supplemented with a simple approximate calculation to explain this rescaling. A similar approach was followed in Ref. 8 to explain both the rescaling of diffusion coefficients and the appearance of a differential flow among chemical species. The idea that diffusion is rescaled by the interaction with immobile species (buffers) is also widespread in biology. For example, the interaction of calcium ions with buffers ${ }^{9}$ accounts for the dependence of calcium diffusion on calcium concentration. ${ }^{10}$ Thus, it is clear that, given a reaction scheme, it is very useful to find a way to predict the changes in the effective diffusivity of the various species.

In this article we approach the rescaling problem from an analytical point of view. The main feature underlying the ability to produce such rescaling is the existence of at least two time scales in the reaction-diffusion system. In fact, that is the common property of all the systems mentioned before. Therefore, in this article we focus on a two-time scale analysis of reaction-diffusion systems that contain processes which occur on different time scales. The approximate calculations of Refs. 7 and 8 did not take this time scale splitting into account. On the other hand, they were done only for one reaction of the form $U+V \underset{k^{\prime}}{\stackrel{k}{k}} W$ with $V$ and $W$ both immobile species. In this article we show how to handle more general reactions. We also show that the rescaling of diffusion coefficients obtained in Ref. 7 or 8 is reobtained in our setting. Another drawback of the simple approach of Ref. 7 or 8 is the lack of information about the initial conditions for the rescaled equations. These equations give the evolution for an approximation to the concentrations and the initial conditions must be changed accordingly. Our derivation automatically gives information on the new initial conditions. It also shows under which assumptions it is possible to reduce the original dynamical equations and what concentrations and time scales these new equations describe.

We apply our calculations to two particular systems of interest: the Selkov model ${ }^{11}$ and the ferrocyanide-iodidesulfite (FIS) reaction. ${ }^{12}$ The first one is a simple model that is 
able to describe single frequency oscillations in the glycolytic pathway. ${ }^{13}$ Analytical and numerical studies have shown that, in the spatially inhomogeneous case, this model is also able to support Turing patterns, provided that the product and substrate diffuse at different rates. ${ }^{14}$ The FIS reaction, on the other hand was originally developed by Edblom et al. ${ }^{12}$ as an example of a chemical system that can support sustained oscillations. Later experiments ${ }^{15,16}$ have shown a variety of patterns that can be easily visualized since they appear as a variation in the $\mathrm{pH}$. Theoretical explanations of these patterns also require that the various species diffuse at different rates. ${ }^{17,18}$ Thus, in both of these cases it is important to determine whether the necessary difference in the diffusion coefficients can be produced by the interactions with immobile species that occur on a fast time scale. This is the point we try to unveil using the procedure described in this article.

The organization of the article is as follows. In Sec. II we briefly review the analytic tools to study two time scale systems. In Sec. III, IV, and V we show how to handle reaction-diffusion systems of this sort under different assumptions. The case of the Selkov model is described in Sec. VI and that of the FIS reaction in Sec. VII. Finally, the conclusions are summarized in Sec. VIII.

\section{THE EXISTENCE OF VARIOUS TIME SCALES AND THE POSSIBILITY OF REDUCING THE NUMBER OF VARIABLES}

Many natural systems involve multiple processes that occur at different rates. Often such systems are well described by just two time scales. Then a small parameter can be introduced, which is given by the ratio between the two time scales. When there is a small parameter, the analysis usually relies on perturbative methods. This occurs, for example, in Hamiltonian systems that are "almost" integrable. In that case an averaging method is used (see, e.g., Ref. 19, p. 167), which results in a reduction of the number of equations we have to deal with. Adiabatic invariants are obtained in this way. Another situation in which there is a time scale which is much slower than the rest takes place near local bifurcations. Exactly at the bifurcation parameter value there is a center manifold on which the evolution is governed by small nonlinear terms, while the contraction or expansion along the stable and unstable manifolds, respectively, is ruled by larger linear terms. In that case a systematic expansion can also be introduced to obtain the evolution on the center manifold. This also involves a reduction in the number of evolution equations, since this manifold is of lower dimension than the whole phase space (see, e.g., Ref. 19, p. 117). This approach can also be used near the bifurcation point. Similar in spirit, but not tied to any type of bifurcation, is the projection onto an inertial manifold for dissipative partial differential equations (PDEs) (see, e.g., Ref. 20). When it exists, the long-term evolution of the infinite dimensional dynamical system effectively occurs on the (finite dimensional) inertial manifold. This allows a reduction from the PDEs to a finite set of ordinary differential equations (ODEs).
The reduction to a center or inertial manifold is achieved by replacing some of the original differential equations by algebraic relations. This is called adiabatic elimination. In doing this, there is almost a one-to-one relationship between time scales and variables in the following sense. It is possible to rewrite the original equations so that only a subset of variables evolves on the fast scale. Then, as many differential equations as "fast" variables can be replaced by algebraic relations. The fast variables very rapidly adjust themselves to the variation of the "slow" variables whose evolution is described by differential equations. Introducing the right rescalings in order to separate the variables in this way is the first step in the analysis of chemical systems under the quasisteady state assumption, ${ }^{21,22}$ which has been largely used when enzymatic reactions are involved. ${ }^{2}$ However, there is another approach very well suited to situations in which there is not a clear separation between fast and slow variables, even if there are processes occurring on different time scales. This is the method we use in Secs. III and IV and is called multiple scales. ${ }^{23}$

\section{THE CASE OF FAST REVERSIBLE REACTIONS}

In this section we describe the main results that are obtained in the case of fast reversible reactions using the method of multiple scales. Their detailed derivation is included in a set of accompanying notes. ${ }^{24} \mathrm{We}$ consider a system of $n+N+1$ components that react and diffuse and assume that there are two groups of time scales in the system: fast and slow. We assume that diffusion and the external feed or removal of the species occur on the slow time scale, while there is a subgroup of reactions (we work out the details for the case of one) that occur on the rapid time scale. There are $n+1$ species $\left(S_{1}, \ldots, S_{n}\right.$, and $\left.Q\right)$ involved in the fast reactions, while there are other $N\left(V_{1}, \ldots, V_{N}\right.$, where $N$ might equal zero) that are not. The spatio-temporal variation of the various concentrations is described by a set of $n+N+1$ reaction-diffusion equations of the form:

$$
\begin{aligned}
\frac{\partial s_{i}}{\partial t} & =\frac{f_{i}}{\epsilon}+g_{s i}+D_{s i} \nabla^{2} s_{i}, 1 \leqslant i \leqslant n, \\
\frac{\partial q}{\partial t} & =\frac{f_{q}}{\epsilon}+g_{q}+D_{q} \nabla^{2} q, \\
\frac{\partial \mathrm{v}_{i}}{\partial t} & =g_{\mathrm{v} i}+D_{\mathrm{v} i} \nabla^{2} \mathrm{v}_{i}, 1 \leqslant i \leqslant N,
\end{aligned}
$$

which are to be solved subject to boundary and initial conditions for $s_{i}(\mathbf{x}, t), q(\mathbf{x}, t)$, and $\mathrm{v}_{i}(\mathbf{x}, t)$. In Eqs. (1)-(3), $s_{i}(\mathbf{x}, t), q(\mathbf{x}, t), \mathbf{v}_{i}(\mathbf{x}, t), D_{s i}, D_{q}$, and $D_{\mathrm{v} i}$ are the concentrations and diffusion coefficients of the $n+N+1$ species, $S_{i}(1 \leqslant i \leqslant n), Q$, and $V_{i}(1 \leqslant i \leqslant N)$, respectively. $f_{i}, f_{q}, g_{s i}, g_{q}$, and $g_{\mathrm{v} i}$ are functions of the concentrations: $f_{i}$ and $f_{q}$ do not depend on $\mathrm{v}_{1}, \ldots, \mathrm{v}_{N}$, but $g_{s i}, g_{q}$, and $g_{\mathrm{v} i}$ may depend on any of the concentrations, including $\mathrm{v}_{1}, \ldots, \mathrm{v}_{N}$. These functions model the variation of the concentrations due to the chemical reactions and to the external feed or removal of each species. The $f$ terms only include the fast reactions while both the slow reactions, the feed, and the removal are included in the $g$ terms. We make the difference between the two time scales explicit by introducing the small 
parameter, $\epsilon$, which is of the order of the ratio between the time scales. $Q$ is any species involved in the fast reaction whose concentration varies because of it (see later). In the case with more fast reactions, we separate as many species, $Q_{i}$, as fast reactions, whenever that is possible.

We are interested only in the variations that occur on the slow time scale. As we will show, this slow evolution is described by a reduced set of differential equations. The differential equations that are "eliminated" are actually replaced by algebraic relations among the concentrations. In the case of one fast reaction the system is finally described by $n+N$ differential equations and one algebraic relation of the form $Q=Q\left(s_{1}, \ldots, s_{n}\right)$, instead of the original set, Eqs. (1)-(3). In the general case of $l$ fast reactions $(l \geqslant 1)$, up to $l$ differential equations can be replaced by algebraic relations (provided that the number of species involved in the fast reactions is larger than $l$ ). As mentioned before, in this article we only work out in detail the case of one fast reaction. Under certain assumptions, the case of more than one fast reaction can be handled in a similar way.

We now consider that there is only one fast reaction, which we write as

$$
\sum_{i=1}^{n} \alpha_{i} S_{i}+\alpha_{q} Q \underset{k^{\prime} / \epsilon}{\rightleftharpoons / \epsilon} \sum_{i=1}^{n} \beta_{i} S_{i}+\beta_{q} Q,
$$

where $\alpha_{j}$ and $\beta_{j}$ stand for the stoichiometric coefficients of the reactants and products, respectively. Here we are assuming that the reaction occurs much faster in both directions than any other process in the system. We make this explicit by writing the reaction rates as $k / \epsilon$ and $k^{\prime} / \epsilon$, with $k$ and $k^{\prime}$ of order one. As usual, we describe the chemical reaction by mass action kinetic equations. ${ }^{25}$ Thus the terms with $f_{i}$ and $f_{q}$ in Eqs. (1)-(2) can be written as

$$
\begin{aligned}
\frac{f_{i}}{\epsilon}\left(s_{1}, \ldots, s_{n}, q\right)= & \left(\beta_{i}-\alpha_{i}\right)\left(\frac{k}{\epsilon}\left(\prod_{j=1}^{n} s_{j}^{\alpha_{j}}\right) q^{\alpha_{q}}\right. \\
& \left.-\frac{k^{\prime}}{\epsilon}\left(\prod_{j=1}^{n} s_{j}^{\beta_{j}}\right) q^{\beta_{q}}\right), \\
\frac{f_{q}}{\epsilon}\left(s_{1}, \ldots, s_{n}, q\right)= & \left(\beta_{q}-\alpha_{q}\right)\left(\frac{k}{\epsilon}\left(\prod_{j=1}^{n} s_{j}^{\alpha_{j}}\right) q^{\alpha_{q}}\right. \\
& \left.-\frac{k^{\prime}}{\epsilon}\left(\prod_{j=1}^{n} s_{j}^{\beta_{j}}\right) q^{\beta_{q}}\right) .
\end{aligned}
$$

The functions $f_{i}$ and $f_{q}$ are then related by

$$
\left(\beta_{q}-\alpha_{q}\right) f_{i}=\left(\beta_{i}-\alpha_{i}\right) f_{q} .
$$

The condition on $Q$ mentioned before is equivalent to saying that $\beta_{q}-\alpha_{q} \neq 0$. In this way, as required, the concentration of $Q$ varies due to the fast reaction.

We then define two times that take into account the two groups of time scales present in the problem, the fast and the slow time scale, respectively, $T \equiv t / \epsilon$ and $\tau \equiv t$. We introduce this rescaling explicitly so that both $T$ and $\tau$ are quantities of order one. We then assume that the concentrations of the species involved in the fast reaction depend on these two time variables, $s_{i}=s_{i}(T, \tau), q=q(T, \tau),{ }^{26}$ while the rest of the concentrations, $\mathrm{v}_{i}, 1 \leqslant i \leqslant N$, only depend on the slow

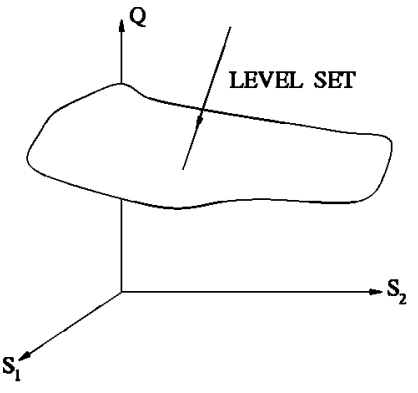

(a)

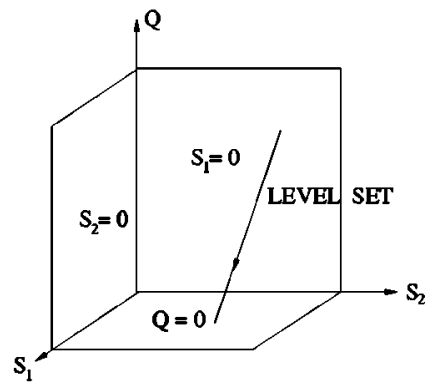

(b)
FIG. 1. Integration of the fast equations for $n=2$. The straight line is the level set which is uniquely determined by the values of the (in this case two) constants of motion. The arrow indicates how the system evolves in time. Each level set intersects the manifolds of fixed points of the fast equations at isolated points. The first point of intersection provides the initial condition for the reduced (slow) evolution equations. (a) The case of one fast reversible reaction. (b) The case of one fast irreversible reaction.

time variable, $\tau$. Following the steps described in Ref. 24 we find that, on the fast time scale, the concentrations approach slowly varying functions of time $\sigma_{i}$ and $\theta$ :

$$
\begin{aligned}
& s_{i} \approx \sigma_{i}(\tau)+\mathcal{O}(\epsilon), 1 \leqslant i \leqslant n, \\
& q \approx \theta(\tau)+\mathcal{O}(\epsilon),
\end{aligned}
$$

where

$$
\theta=\left(\frac{k^{\prime}}{k}\right)^{1 /\left(\alpha_{q}-\beta_{q}\right)} \prod_{j=1}^{n} \sigma_{j}^{\left(\beta_{j}-\alpha_{j}\right) /\left(\alpha_{q}-\beta_{q}\right)},
$$

and

$$
\begin{aligned}
(1+ & \left.\left(\frac{\beta_{i}-\alpha_{i}}{\beta_{q}-\alpha_{q}}\right)^{2} \frac{\theta}{\sigma_{i}}\right) \frac{\partial \sigma_{i}}{\partial \tau}+\frac{\beta_{i}-\alpha_{i}}{\beta_{q}-\alpha_{q}} \sum_{j \neq i} \frac{\beta_{j}-\alpha_{j}}{\beta_{q}-\alpha_{q}} \frac{\theta}{\sigma_{j}} \frac{\partial \sigma_{j}}{\partial \tau} \\
= & D_{s i} \nabla^{2} \sigma_{i}+g_{s i}\left(\left\{\sigma_{j}\right\}, \theta,\left\{\mathrm{v}_{k}\right\}\right) \\
& -\frac{\beta_{i}-\alpha_{i}}{\beta_{q}-\alpha_{q}}\left(g_{q}\left(\left\{\sigma_{j}\right\}, \theta,\left\{\mathrm{v}_{k}\right\}\right)+D_{q} \nabla^{2} \theta\right) .
\end{aligned}
$$

Equations (10)-(11) together with Eq. (3) [where $g_{\mathrm{v} i}$ $\left.=g_{\mathrm{v} i}\left(\left\{\sigma_{j}\right\}, \theta,\left\{\mathbf{v}_{k}\right\}\right)\right]$ constitute the reduction of the original set, Eqs. (1)-(3). Clearly, this new set of equations describes the evolution on the slow time scale. Given initial conditions $s_{i}(t=0), q(t=0)$, and $\mathrm{v}_{i}(t=0)$ for Eqs. (1)-(3), the initial conditions for Eq. (11) can be uniquely determined: they are the asymptotic values that the solutions of the fast equations approach for the given $s_{i}(t=0), q(t=0)$, and $\mathrm{v}_{i}(t=0)$. As explained in Ref. 24 , the fast equations have so many constants of motion that the evolution takes place on a onedimensional level set defined by this constant. In this way it is straightforward to obtain the corresponding initial condition, as is schematically depicted in Fig. 1(a) for the $n=2$ case.

The meaning of the reduction is the following. After a very short transient (during which the fast reaction equilibrates) the dynamics is restricted to a lower-dimensional manifold on which the evolution is slow. This manifold is defined by the algebraic constraint, Eq. (10). We illustrate this schematically in Fig. 2(a). The separation into a "fast" 


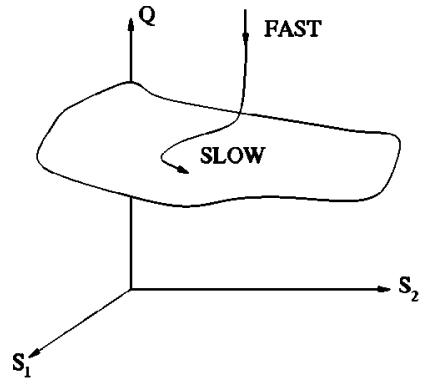

(a)

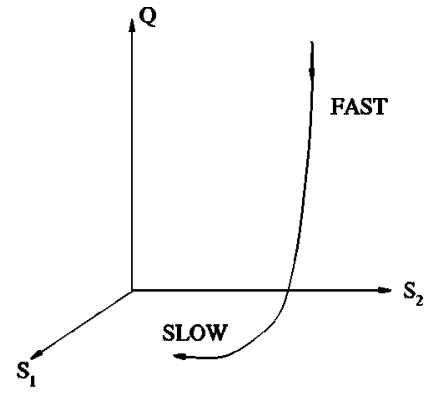

(b)
FIG. 2. Schematic illustration of what the reduction means in the case of one fast reversible reaction (a) and in the case of an irreversible one (b). In both cases, the reduced equations describe the evolution on the (slow) manifold that is approached after a fast transient.

and a "slow" process is possible because of the different weights that the various terms have in Eqs. (1) and (2). It is clear that the order-of-magnitude of the fast reaction terms in these equations not only depends on the constants, $k / \epsilon$ and $k^{\prime} / \epsilon$, but also on the concentrations of the variables involved. The calculation we have presented is consistent as long as there are not very large differences in the concentrations so that the fast terms, $f_{i} / \epsilon$ and $f_{q} / \epsilon$, are effectively of the same order-of-magnitude as the slow ones (e.g., $g_{s i}$ ). If this happens after a transient, as a consequence of the evolution, the calculation is still consistent. But if the initial conditions are such that, at $t=0, f_{i} / \epsilon \sim g_{s i}$ or $f_{q} / \epsilon \sim g_{q}$, then the concentrations should be rescaled accordingly from the very beginning. This is what happens, for example, in most systems involving enzymatic reactions. The (spatially) homogeneous dynamics of this type of system has been mostly studied in the quasisteady-state assumption. ${ }^{21,22} \mathrm{~A}$ very nice description of how the different variables need to be rescaled in some of these cases can be found in Ref. 22. We also discuss this later in Secs. V and VI. In this section we assume that, at least initially, all fast terms are larger than the slow ones.

We now consider a particular case of interest for applications: the one in which three species are involved in the fast reaction, two of which do not diffuse. For example, this is the case in which a complex is formed out of two species and the complex and one of its forming species do not diffuse. This is only a particular case of our general setting and it is convenient to use as our species $Q$ either one of the nondiffusing species. So, we have $S_{1}, S_{2}$, and $Q$, and $D_{s 2}$ $=D_{q}=0$. For simplicity, from now on we will not write the dependence of $g_{s i}$ and $g_{q}$ on the concentrations explicitly. After some algebraic manipulation, we obtain from the corresponding Eqs. (10) and (11) (see Ref. 24)

$$
\theta=\left(\frac{k^{\prime}}{k}\right)^{1 /\left(\alpha_{q}-\beta_{q}\right)} \sigma_{1}^{\left(\beta_{1}-\alpha_{1}\right) /\left(\alpha_{q}-\beta_{q}\right)} \sigma_{2}^{\left(\beta_{2}-\alpha_{2}\right) /\left(\alpha_{q}-\beta_{q}\right)},
$$

$$
\begin{aligned}
\frac{\partial \sigma_{1}}{\partial \tau}= & \frac{A_{22}}{\Delta} D_{s 1} \nabla^{2} \sigma_{1}+\frac{A_{22}}{\Delta} g_{s 1}-\frac{A_{12}}{\Delta} g_{s 2} \\
& +\frac{A_{12}\left(\beta_{2}-\alpha_{2}\right)-A_{22}\left(\beta_{1}-\alpha_{1}\right)}{\Delta\left(\beta_{q}-\alpha_{q}\right)} g_{q},
\end{aligned}
$$

where

$$
\begin{aligned}
& A_{11}=1+\left(\frac{\beta_{1}-\alpha_{1}}{\beta_{q}-\alpha_{q}}\right)^{2} \frac{\theta}{\sigma_{1}}, A_{12}=\frac{\left(\beta_{1}-\alpha_{1}\right)\left(\beta_{2}-\alpha_{2}\right)}{\left(\beta_{q}-\alpha_{q}\right)^{2}} \frac{\theta}{\sigma_{2}}, \\
& A_{21}=\frac{\left(\beta_{1}-\alpha_{1}\right)\left(\beta_{2}-\alpha_{2}\right)}{\left(\beta_{q}-\alpha_{q}\right)^{2}} \frac{\theta}{\sigma_{1}}, A_{22}=1+\left(\frac{\beta_{2}-\alpha_{2}}{\beta_{q}-\alpha_{q}}\right)^{2} \frac{\theta}{\sigma_{2}},
\end{aligned}
$$

and $\Delta=A_{22} A_{11}-A_{21} A_{12}$. In this case, the reduction introduces a rescaling of the diffusion coefficient, such that the new coefficient, $D_{s 1}^{\prime}$, is related to the original one, $D_{s 1}$ by

$$
\begin{aligned}
D_{s 1}^{\prime}= & \frac{A_{22}}{\Delta} D_{s 1} \\
= & \frac{\left(\beta_{q}-\alpha_{q}\right)^{2} \sigma_{1} \sigma_{2}+\left(\beta_{2}-\alpha_{2}\right)^{2} \theta \sigma_{1}}{\left(\beta_{q}-\alpha_{q}\right)^{2} \sigma_{1} \sigma_{2}+\left(\beta_{1}-\alpha_{1}\right)^{2} \theta \sigma_{2}+\left(\beta_{2}-\alpha_{2}\right)^{2} \theta \sigma_{1}} \\
& \times D_{s 1} .
\end{aligned}
$$

In certain cases there are conserved quantities in the system that allow us to write $\sigma_{2}$ as a function of $\sigma_{1}$, in which case it is then possible to write the rescaled diffusion coefficient, $D_{s 1}^{\prime}$, only as a function of $\sigma_{1}$. In particular, this is the case for the situation previously discussed in the literature in which the fast reaction is of the form $S_{1}+S_{2} \underset{k^{\prime} / \epsilon}{\stackrel{k / \epsilon}{\rightleftharpoons}} Q$. In this case the quantity $s_{2}+q$ remains constant during the evolution, from which we may conclude that $\sigma_{2}+\theta$ also remains constant. Setting $C=\sigma_{2}+\theta$ and using Eq. (12), which in this case reads $\theta=\left(k / k^{\prime}\right) \sigma_{1} \sigma_{2}$, we obtain that the rescaled diffusion coefficient, Eq. (15) can be rewritten as

$$
D_{s 1}^{\prime}=\frac{\left(1+\left(k / k^{\prime}\right) \sigma_{1}\right)^{2}}{\left(1+\left(k / k^{\prime}\right) \sigma_{1}\right)^{2}+\left(k / k^{\prime}\right) C} D_{s 1},
$$

which, in the limit of $\left(k / k^{\prime}\right) \sigma_{1} \ll 1$ reduces to the values obtained in Refs. 7 or 8 . Notice that this rescaled coefficient depends on the dissociation constant $k_{d} \equiv k^{\prime} / k$ as in Ref. 9. It is interesting to note that, as expected, in the limit of $\sigma_{1}$ $\rightarrow \infty$, Eq. (15) implies that $D_{s 1}^{\prime} \rightarrow D_{s 1}$. This may be understood in the following way. If $S_{1}$ is in excess, there is not enough $S_{2}$ to trap $S_{1}$ particles. Thus, most of the $S_{1}$ particles diffuse with their "normal" diffusion coefficient, $D_{s 1}$. In particular, this agrees with both the experimental measurements of calcium diffusion in the presence of buffers and the qualitative explanation of this behavior. ${ }^{10}$ Expression (14) also implies that $D_{s 1}^{\prime} \rightarrow D_{s 1}$ as $\sigma_{1} \rightarrow \infty$.

In the more general case in which at least two of the species involved in the fast reaction diffuse, the reduction not only rescales the diffusion coefficients, but also introduces cross-diffusion terms. Consider for example the case we have just analyzed, but suppose that $D_{s 2} \neq 0$. Then, from Eq. (11) we get:

$$
\begin{aligned}
\frac{\partial \sigma_{1}}{\partial \tau}= & \frac{A_{22}}{\Delta} D_{s 1} \nabla^{2} \sigma_{1}-\frac{A_{12}}{\Delta} \nabla^{2} \sigma_{2}+\frac{A_{22}}{\Delta} g_{s 1}-\frac{A_{12}}{\Delta} g_{s 2} \\
& +\frac{A_{12}\left(\beta_{2}-\alpha_{2}\right)-A_{22}\left(\beta_{1}-\alpha_{1}\right)}{\Delta\left(\beta_{q}-\alpha_{q}\right)} g_{q},
\end{aligned}
$$




$$
\begin{aligned}
\frac{\partial \sigma_{2}}{\partial \tau}= & \frac{A_{11}}{\Delta} D_{s 2} \nabla^{2} \sigma_{2}-\frac{A_{21}}{\Delta} \nabla^{2} \sigma_{1}+\frac{A_{11}}{\Delta} g_{s 2}-\frac{A_{21}}{\Delta} g_{s 1} \\
& +\frac{A_{21}\left(\beta_{1}-\alpha_{1}\right)-A_{11}\left(\beta_{2}-\alpha_{2}\right)}{\Delta\left(\beta_{q}-\alpha_{q}\right)} g_{q},
\end{aligned}
$$

with $A_{i j}$ and $\Delta$ defined as before. If the factors of the form $A_{i j} / \Delta$ do not vary too much with time or space and can be treated as constants, then it is possible to find linear combinations of $\sigma_{1}$ and $\sigma_{2}, \quad \psi=a_{1} \sigma_{1}+a_{2} \sigma_{2}$ and $\xi=b_{1} \sigma_{1}$ $+b_{2} \sigma_{2}$, such that their evolution equations are of the form:

$$
\begin{aligned}
& \frac{\partial \psi}{\partial \tau}=D_{\psi} \nabla^{2} \psi+g_{\psi}, \\
& \frac{\partial \xi}{\partial \tau}=D_{\xi} \nabla^{2} \xi+g_{\xi} .
\end{aligned}
$$

This two-variable reaction-diffusion system can be analyzed in terms of the usual inhibitor-activator scheme. However, none of the quantities, $\psi$ or $\xi$, correspond to the concentration of any actual chemical species present in the system.

In certain cases, a similar (but more complicated) calculation can be done if there is more than one fast reaction. Depending on the number of fast reactions and on the number of species involved, as many variables as fast reactions can be eliminated by this procedure. However, this is not always possible to accomplish and the situation has to be analyzed on a one-by-one basis. In any case, these systems may be handled in an easier way following the approach of Sec. V, of which we show several examples in the subsequent sections.

\section{THE CASE OF ONE FAST IRREVERSIBLE REACTION}

The case of fast reversible reactions can be clearly handled in the way described in the previous section. In the case that some of the reactions are fast in one direction and slow in the reverse one, or if it only occurs in one direction, then it is not always possible to find an algebraic relation like Eq. (10). In general, the system relaxes rapidly to a situation in which the concentration of one of the variables is almost negligible. Therefore, the reduction is not related to the existence of an algebraic relation of the form Eq. (10) but rather to the possibility of neglecting one of the concentrations. In this section we analyze the simplest such example: the case of one fast irreversible reaction. We illustrate the corresponding behavior schematically in Fig. 2(b).

As in the previous section, we consider a system described by Eqs. (1)-(3) where now $f_{i}$ and $f_{q}$ are given by Eqs. (5) and (6) with $k^{\prime}=0$. Following the steps of Ref. 24, we find that one of the species, $Q$, approaches very fast a negligible value $\left(q \approx \epsilon q^{(1)}\right)$, while the others approach slowly varying amplitudes, $\sigma_{i}\left(s_{i} \approx \sigma_{i}(\tau)+\mathcal{O}(\epsilon)\right)$. In this situation it is not always possible to perform a two-time scale analysis as before. In fact, we can only find reduced equations if $\alpha_{q}=1$, in which case we get

$$
\begin{aligned}
\frac{\partial \sigma_{i}}{\partial \tau}= & g_{s i}\left(\left\{\sigma_{j}\right\}, 0,\left\{\mathrm{v}_{k}\right\}\right)-\frac{\beta_{i}-\alpha_{i}}{\beta_{q}-\alpha_{q}} g_{q}\left(\left\{\sigma_{j}\right\}, 0,\left\{\mathrm{v}_{k}\right\}\right) \\
& +D_{s i} \nabla^{2} \sigma_{i},
\end{aligned}
$$

or if $\alpha_{q}>1$ and $g_{q}\left(\left\{\sigma_{j}\right\}, 0,\left\{\mathbf{v}_{k}\right\}\right)=0$, in which case we get

$$
\frac{\partial \sigma_{i}}{\partial \tau}=g_{s i}\left(\left\{\sigma_{j}\right\}, 0,\left\{\mathbf{v}_{k}\right\}\right)+D_{s i} \nabla^{2} \sigma_{i} .
$$

In none of these cases do the diffusion coefficients get rescaled. This can be intuitively understood from the microscopic point of view. Consider for example species $S_{1}$ which is irreversibly transformed into other species under the fast reaction. Then, the only particles of species $S_{1}$ that are still in the system are those that have not undergone the fast reaction. Those particles diffuse with their normal diffusion coefficient. The initial conditions for the reduced equations can be obtained as in the case of the previous section. This is shown schematically in Fig. 1(b).

\section{ANOTHER APPROACH TO REDUCTION}

In Sec. III we performed a systematic two-time scale expansion of the original equations of motion and obtained a reduced set of equations. The reduction was a consequence of the algebraic relation Eq. (10) and of the ability to separate the dependence on the two time scales in a certain way. In this section we start seeking an algebraic relation among the concentrations from the very beginning, without making precise statements on the time-dependence of the variables we are going to keep (the $s_{i}$ of Sec. III). In some sense, this is almost an extension of the quasisteady-state approximation $^{22}$ for partial differential equations. However, we do not discuss how the various variables need to scale with the small parameter of the system in order to start a consistent calculation. Rather, we look for an algebraic equation in the original variables. Under the assumptions of Sec. III, this new calculation gives the same result as before. In other situations, an a posteriori check is necessary in order to guarantee the validity of the reduction.

As before, we assume that there is only one fast reaction and that we want to "eliminate" one of the variables, $q$, involved in that reaction. To this end we expand

$$
q \approx q^{(0)}\left(s_{1}, \ldots, s_{n}\right)+\epsilon q^{(1)}\left(s_{1}, \ldots, s_{n}\right),
$$

and replace it in Eq. (2). We find

$$
\begin{aligned}
\sum_{i=1}^{n} & \frac{\partial q^{(0)}}{\partial s_{i}} \frac{\partial s_{i}}{\partial t}+\epsilon \sum_{i=1}^{n} \frac{\partial q^{(1)}}{\partial s_{i}} \frac{\partial s_{i}}{\partial t} \\
= & \frac{f_{q}\left(q^{(0)}, s_{1}, \ldots, s_{n}\right)}{\epsilon}+\frac{\partial f_{q}}{\partial q} q^{(1)} \\
& \quad+{ }_{q}\left(q^{(0)}, s_{1}, \ldots, s_{n}, \mathrm{\vee}_{1}, \ldots, \mathrm{v}_{N}\right)+D_{q} \nabla^{2} q^{(0)}+\mathcal{O}(\epsilon)
\end{aligned}
$$

If we introduce the two time scales, $T$ and $\tau$ as before, then

$$
\frac{\partial s_{i}}{\partial t}=\frac{1}{\epsilon} \frac{\partial s_{i}}{\partial T}+\frac{\partial s_{i}}{\partial \tau}
$$


In the previous section we could justify that $s_{i}=\zeta_{i}(T, \tau)$ $+\sigma_{i}(\tau)$ with $\zeta_{i} \ll \sigma_{i}$ for $T$ big enough. This condition implies that $\partial s_{i} / \partial t \approx \partial s_{i} / \partial \tau$, so that $\partial s_{i} / \partial t \sim \mathcal{O}(1)$. If we assume this, then, equating terms with equal powers of $\epsilon$ in Eq. (23) we obtain

$$
\begin{aligned}
f_{q}\left(q^{(0)}, s_{1}, \ldots, s_{n}\right)=0 & \\
q^{(1)}= & \frac{1}{\partial f_{q} / \partial q}\left(\sum_{i=1}^{n} \frac{\partial q^{(0)}}{\partial s_{i}} \frac{\partial s_{i}}{\partial t}\right. \\
& \left.\quad-g_{q}\left(q^{(0)}, s_{1}, \ldots, s_{n}, \mathrm{v}_{1}, \ldots, \mathrm{v}_{N}\right)-D_{q} \nabla^{2} q^{(0)}\right),
\end{aligned}
$$

provided that $\partial f_{q} / \partial q \neq 0$. Clearly, it is not possible to do this in the case of one fast irreversible reaction. In most cases, from Eq. (24) we obtain the desired algebraic relationship $q^{(0)}=q^{(0)}\left(s_{1}, \ldots, s_{n}\right)$. Replacing this relationship in Eq. (25) we obtain $q^{(1)}$ as a function of $s_{1}, \ldots, s_{n}$ and $\mathrm{v}_{1}, \ldots, \mathrm{v}_{N}$. Replacing these functions in the expansion (22) and inserting it in the equations for the concentrations $s_{i}$ we get Eq. (11) with $s_{i}$, instead of $\sigma_{i}$ and $q^{(0)}$ instead of $\theta$.

Even if we cannot guarantee a priori that $\partial s_{i} / \partial t$ $\approx \partial s_{i} / \partial \tau$, we can perform this calculation and check afterwards if it is consistent. Namely, if the values of $q^{(0)}$ and $q^{(1)}$ that we obtain satisfy

$$
\left|\epsilon q^{(1)}\right| \ll\left|q^{(0)}\right|
$$

then the calculation is correct. Notice that if the fast variation of some $s_{i}$ is relevant, then $q^{(1)}$ can be of order $1 / \epsilon$ and the condition Eq. (26) will fail. Under the assumptions of Sec. III (fast reversible reactions), this "new" calculation gives exactly the same result. If some of these assumptions do not hold, we can still follow this new calculation and then perform the check we have just mentioned. For this reason, it may be extended to cases not included in the assumptions of Sec. III. However, the validity of this approach can only be checked a posteriori. On the other hand, it is not clear how to obtain the initial conditions for the reduced equations. We discuss this in detail in Ref. 24.

\section{THE SELKOV MODEL}

The Selkov model ${ }^{11}$ represents an open monosubstrate and monoproduct reaction catalized by an allosteric ${ }^{27}$ enzyme which is inhibited by the substrate and activated by the product. The model describes the kinetics of the reactions involved in the appearance of a single frequency oscillation in the glycolytic pathway. ${ }^{13}$ The allosteric properties of the enzyme (phosphofructokinase, PFK) are taken into account in an approximate way, ${ }^{28}$ through a factor $(\gamma)$ which represents both the degree of product activation and that of substrate inhibition. What makes the PFK special is that it belongs to a path that globally produces adenosine triphosphate (ATP) but in the specific step it catalizes, it produces adenosine diphosphate (ADP) out of ATP. Given that the PFK is regulated by its global behavior, there is a positive feedback exerted on the PFK by the ADP. This feedback gives rise to the appearance of self oscillations on the concentration of ATP and ADP.
As shown by Selkov, ${ }^{11}$ the time evolution of the dynamical system that represents the chemical reactions of an ideally homogeneous medium can be confined on a twodimensional manifold given by the ATP and ADP concentrations. He then showed that the frequency of the limit cycle predicted by his model is very close to the one found in experiments. Moreover, it is simple to show that if spatial variations are permitted, then Turing instabilities can be observed in this system provided that ATP diffuses more slowly than ADP. ${ }^{14}$ The possibility of finding mesoscopic chemical patterns in biological cells is of fundamental importance. Many other chemical species, not necessarily involved in this reaction, could rest on ATP-rich paths created by this instability. Besides, as pointed out by Goldbeter, ${ }^{28}$ if the cell membrane is permeable to chemical reactants a propagating structure may arise from the coupling of several neighboring cells possesing enzyme oscillators synchronized by the source of the substrate. It is also believed that this kind of chemical supracellular pattern could play a role in embryonic development since the dimensions for which they arise are those of the morphogenetic field. However, at least one point against this possibility must be overcome for glycolytic spatial patterns to be observed in cells: as mentioned in the introduction, Turing patterns are mainly driven by differences in the diffusion coefficients of chemical species. Thus in this case the diffusion coefficient of substrate $S_{1}$ (ATP) and the product $S_{2}$ (ADP) should become significantly different for the structures to be observed. But, as long as these molecules have almost the same structure and mass, it is clear that without a selective interaction with nondiffusing species, the necessary difference in the magnitude of the diffusion coefficients cannot be achieved. It would be interesting to apply in this case the methods developed in the last sections and study if the rescaling of the diffusion coefficients can be large enough for this kind of pattern to be observed.

The simple kinetic model developed by Selkov reads:

$$
\begin{gathered}
\mathrm{v}_{1} S_{1}+E S_{k_{-1} / \epsilon}^{k_{+1} / \epsilon} S_{1} E S_{2}^{\gamma}, \\
S_{1} E S_{2}^{\gamma} \longrightarrow E S_{2}^{\gamma}+S_{2} \longrightarrow, \\
\mathrm{v}_{2} \rightarrow \epsilon \\
\gamma S_{2}+E \underset{k_{-3} / \epsilon}{\rightleftharpoons} E S_{2}^{\gamma},
\end{gathered}
$$

where $S_{1}$ is supplied by an external source at the rate $\mathrm{v}_{1}$ which is supposed to remain constant during the reaction. From the equations it is clear that $S_{1}$ is irreversibly converted into the product molecules $S_{2}$. The product is then removed by an irreversible sink at the rate $\mathrm{v}_{2}$. The free enzyme $E$ is inactive unless when it has $\gamma$ product molecules bounded, forming the complex $E S_{2}^{\gamma}$. We start from the corresponding Eqs. (1)-(3) in which we assume that the sink of the product behaves as a first-order reaction $\left(\mathrm{v}_{2}=k_{2} s_{2}\right)$ and that the substrate and product diffuse in space, but neglect the diffusion of the enzymes and its complexes. These are five reactiondiffusion equations for the concentrations $s_{1}=\left[S_{1}\right], s_{2}$ $=\left[S_{2}\right], q_{1}=\left[E S_{2}^{\gamma}\right], \quad q_{2}=\left[S_{1} E S_{2}^{\gamma}\right]$, and $q_{3}=[E]$ (see Ref. 
24). However, taking into account the constancy of the total amount of enzyme $q_{1}(t)+q_{2}(t)+q_{3}(t)=$ const $\equiv e_{0}$ it is possible to reduce the number of equations by one.

According to Selkov's data, the concentration of $E$ and its complexes is three to four orders-of-magnitude below those of ATP and ADP. ${ }^{11}$ So, we can rescale the $q_{i}$ concentrations by a small parameter (of the order of $\epsilon$ ), as done in Ref. 22. In order to discuss the validity of the approximation in different limits we will introduce a new parameter, $\tilde{\epsilon}$, such that $q_{i}=\tilde{\epsilon} \widetilde{q}_{i}$ and $e_{0}=\tilde{\epsilon} \tilde{e}_{0}$, and use the expansion $q_{i}=\tilde{\epsilon} \tilde{q}_{i}$ $=\tilde{\epsilon}\left(q_{i}^{(0)}+\epsilon q_{i}^{(1)}\right)$ to simplify the notation. Thus, $\tilde{\boldsymbol{\epsilon}}$ is proportional to the ratio of enzyme to product or substrate concentration.

We proceed as described in the last section in order to get rid of $\tilde{q}_{1}, \tilde{q}_{2}$, and $\tilde{q}_{3}$ in favor of $s_{1}$ and $s_{2}$. After a lengthy calculation we obtain: ${ }^{24}$

$$
\begin{aligned}
\frac{\partial \xi_{1}}{\partial t}= & \frac{a^{(0)}+\tilde{\epsilon}\left(a^{(1)} b^{(0)}+d^{(1)} a^{(0)}\right)}{\Delta}+\frac{D_{1}}{\Delta}\left(1+\tilde{\epsilon} d^{(1)}\right) \nabla^{2} \xi_{1} \\
& +\tilde{\epsilon} a^{(1)} \frac{D_{2}}{\Delta} \nabla^{2} \xi_{2}, \\
\frac{\partial \xi_{2}}{\partial t}= & \frac{b^{(0)}+\tilde{\epsilon}\left(b^{(1)} a^{(0)}+c^{(1)} b^{(0)}\right)}{\Delta}+\frac{D_{2}}{\Delta}\left(1+\tilde{\epsilon} c^{(1)}\right) \nabla^{2} \xi_{2} \\
& +\tilde{\epsilon} b^{(1)} \frac{D_{1}}{\Delta} \nabla^{2} \xi_{1},
\end{aligned}
$$

where we have defined the rescaled concentrations, $\xi_{1}$ $\equiv k_{+1} /\left(k_{-1}+k_{+2}\right) s_{1} \quad$ and $\quad \xi_{2} \equiv\left(k_{+3} / k_{-3}\right)^{1 / \gamma_{s_{2}}}, \quad \delta \equiv 1$ $+\xi_{2}^{\gamma}\left(1+\xi_{1}\right)$ and

$$
\begin{aligned}
& a^{(0)}=\frac{k_{+1}}{k_{-1}+k_{+2}}\left(\mathrm{v}_{1}-\frac{\tilde{\epsilon} k_{+2} \xi_{1} \xi_{2}^{\gamma}}{\delta} \tilde{e}_{0},\right), \\
& b^{(0)}=\left(\frac{k_{+3}}{k_{-3}}\right)^{1 / \gamma} \frac{\tilde{\epsilon}}{\epsilon} \frac{k_{+2} \xi_{1} \xi_{2}^{\gamma}}{\delta} \tilde{e}_{0}-k_{2} \xi_{2}, \\
& a^{(1)}=\frac{\gamma k_{+1} \xi_{1} \xi_{2}^{\gamma-1} \tilde{e}_{0}}{\left(k_{-1}+k_{+2}\right) \delta^{2}}, \\
& b^{(1)}=\left(\frac{k_{+3}}{k_{-3}}\right)^{1 / \gamma} \frac{\left(k_{-3}-k_{+2}\right) \xi_{2}^{\gamma} \tilde{e}_{0}}{k_{-3} \delta^{2}}, \\
& c^{(1)}=\frac{k_{+1} \xi_{2}^{\gamma} \tilde{e}_{0}}{k_{-3} \delta^{2}}\left(\xi_{1}+\frac{k_{-1}}{k_{-1}+k_{+2}}\right), \\
& d^{(1)}=\left(\frac{k_{+3}}{k_{-3}}\right)^{1 / \gamma} \frac{k_{-3} \gamma\left(1+\xi_{2}^{\gamma}\right) \xi_{2}^{\gamma-1} \tilde{e}_{0}}{\left(k_{-1}+k_{+2}\right) \delta^{2}}, \\
& \Delta=\left(1+\tilde{\epsilon} c^{(1)}\right)\left(1+\tilde{\epsilon} d^{(1)}\right)-\tilde{\epsilon}^{2} a^{(1)} b^{(1)} .
\end{aligned}
$$

First we must notice that both $a^{(0)}$ and $b^{(0)}$ contain terms that are proportional to $\tilde{\epsilon} / \epsilon$ [see Eq. (30)]. Thus, if $\tilde{\epsilon} \sim \boldsymbol{\epsilon}$ $\ll 1$, then the calculation is consistent since we may assume that $\partial \xi_{1} / \partial t$ and $\partial \xi_{2} / \partial t$ are of order one [see Eqs. (28)-
(29)], in which case the conditions $\left|\epsilon q_{i}^{(1)}\right| \ll\left|q_{i}^{(0)}\right|$ are easily satisfied, provided that $\epsilon$ is small enough (see the detailed calculations of Ref. 24). Then, keeping up to linear terms in $\tilde{\epsilon}$, Eqs. (28)-(29) may be approximated by

$$
\begin{aligned}
\frac{\partial \xi_{1}}{\partial t}= & a^{(0)}+\tilde{\epsilon}\left(a^{(1)} b^{(0)}-a^{(0)} c^{(1)}\right) \\
& +D_{1}\left(1-\tilde{\epsilon} c^{(1)}\right) \nabla^{2} \xi_{1}+\tilde{\epsilon} a^{(1)} D_{2} \nabla^{2} \xi_{2}, \\
\frac{\partial \xi_{2}}{\partial t}= & b^{(0)}+\tilde{\epsilon}\left(b^{(1)} a^{(0)}-b^{(0)} d^{(1)}\right) \\
& +D_{2}\left(1-\tilde{\epsilon} d^{(1)}\right) \nabla^{2} \xi_{2}+\tilde{\epsilon} b^{(1)} D_{1} \nabla^{2} \xi_{1} .
\end{aligned}
$$

Also in this approximation there are cross diffusion terms, which can be neglected if $\tilde{\epsilon}$ is small enough. However, under that assumption, the rescaling of the diffusion coefficients is also negligible. The ratio between the effective diffusion coefficients of the substrate and the product is approximately given by $\left(D_{1} / D_{2}\right)\left(1+\tilde{\epsilon} d^{(1)}-\tilde{\epsilon} c^{(1)}\right)$ and this might not give the necessary value to sustain Turing patterns. ${ }^{14}$ Now, the ratio of enzyme to product or substrate concentration (which is proportional to $\tilde{\epsilon}$ in our notation) is very small in experiments done in vitro. It has been argued ${ }^{14}$ that conditions in vivo can be largely different. Namely, if $\tilde{\epsilon} d^{(1)} \sim \mathcal{O}(1)$ or $\tilde{\epsilon} c^{(1)} \sim \mathcal{O}(1)$ then the rescaling could be important. However, the cross-diffusion terms would also be important and a new analysis of pattern formation in this context should be done, which goes beyond the scope of this article. In any case, we must note that the conditions for the reduction to be valid [the natural extension of Eq. (26)] may not hold if $a^{(0)}$ or $b^{(0)}$ become too large due to their dependence on $\tilde{\epsilon} / \epsilon$. This consistency condition must be checked for each specific application before drawing any conclusions for this system. We will study the general reduced system in more detail in the future.

\section{THE FIS REACTION}

We now analyze the case of the FIS reaction, which was originally studied in Ref. 12 and later in Refs. 15, 16, where a variety of patterns were observed. A set of basic reaction steps involved in the FIS system was first proposed in Ref. 12 and then improved in Ref. 29. It was later shown ${ }^{30}$ that, under certain assumptions which hold in the experiments, the evolution may be described in terms of four dynamical variables. We will consider this model, which is given by the following set of reactions:

$$
\begin{gathered}
k_{N_{1} / \epsilon} \\
A+V \underset{k_{N 1}^{\prime} / \epsilon}{\rightleftharpoons} U, \\
k_{N 2} \\
U \rightarrow V, \\
k_{N 3} / \epsilon \\
2 V \rightarrow Z,
\end{gathered}
$$




$$
\begin{aligned}
& \stackrel{k_{N 4} / \epsilon}{Z+U} \rightarrow 3 V, \\
& { }^{k_{N 5}} \\
& Z \rightarrow,
\end{aligned}
$$

where $U=\mathrm{HSO}_{3}^{-}, \mathrm{V}=\mathrm{H}^{+}, \mathrm{A}=\mathrm{SO}_{3}^{2-}$, and $\mathrm{Z}=\mathrm{I}_{2}$. In this case, we start from a set of four reaction-diffusion equations in which we have assumed that the species $V$ and $A$ are fed into the system at the same rate at which all of the species are removed $\left(k_{0}\right)$ and that all the species may diffuse (see Ref. 24).

Taking into account the huge differences in the various rates, Gáspár and Showalter further reduced the corresponding homogeneous dynamical system (our reaction-diffusion equations with no diffusion terms) to a set of two ODEs. With the addition of diffusion terms these two equations form an inhibitor-activator system with bisulfite $(U)$ the inhibitor and protons $(V)$ the activator species. Theoretical analyses and numerical simulations of this type of inhibitoractivator system indicates that the inhibitor needs to diffuse faster than the activator for patterns to be formed. ${ }^{17,18,16}$ This seems unrealistic if protons are the activator species. In this section we apply the methods of Sec. V to see if the diffusion coefficients are rescaled so as to satisfy this theoretical condition.

In order to make this calculation more specific, we consider the parameter values that are used in the experiments when replicating spots are observed: $k_{N 2}=0.06 \mathrm{~s}^{-1} k_{N 1} / \epsilon$ $=5.0 \times 10^{10} \mathrm{M}^{-1} \mathrm{~s}^{-1}, \quad k_{N 1}^{\prime} / \epsilon=8.1 \times 10^{3} \mathrm{~s}^{-1}, \quad k_{N 3} / \epsilon=7.5$ $\times 10^{4} \mathrm{M}^{-1} \mathrm{~s}^{-1}, k_{N 4} / \epsilon=2.3 \times 10^{9} \mathrm{M}^{-1} \mathrm{~s}^{-1}, k_{N 5}=43.68 \mathrm{~s}^{-1}$. It is not completely clear what the values of $\mathrm{v}_{0}$ and $a_{0}$ actually are inside the gel where the reaction takes place. We will consider $a_{0}=0.089$ and $0.0072 \mathrm{M} \leqslant \mathrm{v}_{0} \leqslant 0.085 \mathrm{M}$. We then see why we can introduce the small parameter $\epsilon$ to distinguish between fast and slow reactions. However, we also see that there are more than two time scales. In any case, at this point, we only distinguish two groups. Inspired by the reduction of the ODEs performed in Ref. 30, we seek a reduction of the PDEs in which the variables $a$ and $z$ are eliminated in favor of $u$ and $\mathrm{v}$. To this end we follow the steps of Sec. V considering $S_{1}=U, S_{2}=V, Q_{1}=A$, and $Q_{2}=Z$. Since the $Z$ species is iodine which binds to the gel, its diffusion coefficient may be neglected. For the sake of simplicity, we will also neglect the diffusion term of $A$. With these assumptions we obtain, after a lenghty calculation, ${ }^{24}$

$$
\begin{aligned}
\frac{\partial u}{\partial t}= & -\frac{k_{N 3}}{\epsilon} \frac{\mathrm{v}^{2}}{\Delta}\left(1+\frac{4 k_{N 3} \mathrm{v}}{k_{N 4} u}\right) \\
& +\frac{D_{u}}{\Delta} \nabla^{2} u\left(1+\frac{6 k_{N 3} \mathrm{v}}{k_{N 4} u}+\frac{k_{N 1}^{\prime} u}{k_{N 1} \mathrm{v}^{2}}\right) \\
& +\frac{D_{\mathrm{v}}}{\Delta} \nabla^{2} \mathrm{v}\left(\frac{k_{N 1}^{\prime} u}{k_{N 1} \mathrm{v}^{2}}+\frac{2 k_{N 3} \mathrm{v}}{k_{N 4} u}\right)+B_{u} A_{11}+B_{\mathrm{v}} A_{12},
\end{aligned}
$$

$$
\begin{aligned}
\frac{\partial \mathrm{v}}{\partial t}= & \frac{k_{N 3}}{\epsilon} \frac{\mathrm{v}^{2}}{\Delta}\left(1-\frac{2 k_{N 3} \mathrm{v}^{2}}{k_{N 4} u^{2}}\right) \\
& +\frac{D_{\mathrm{v}}}{\Delta} \nabla^{2} \mathrm{v}\left(1+\frac{k_{N 1}^{\prime}}{k_{N 1} \mathrm{v}}+\frac{k_{N 3} \mathrm{v}^{2}}{k_{N 4} u^{2}}\right) \\
& +\frac{D_{u}}{\Delta} \nabla^{2} u\left(\frac{k_{N 1}^{\prime}}{k_{N 1} \mathrm{v}}+\frac{3 k_{N 3} \mathrm{v}^{2}}{k_{N 4} u^{2}}\right)+B_{\mathrm{v}} A_{22}+B_{u} A_{21},
\end{aligned}
$$

where we have defined $B_{u} \equiv\left(-k_{N 2} u-k_{0} u+k_{0}\left(a_{0}\right.\right.$ $\left.\left.-k_{N 1}^{\prime} u / k_{N 1} \mathrm{v}\right)+\left(k_{N 5}+k_{0}\right)\left(k_{N 3} \mathrm{v}^{2} / k_{N 4} u\right)\right) / \Delta, \quad B_{\mathrm{v}} \equiv\left(k_{N 2} u\right.$ $+k_{0}\left(\mathrm{v}_{0}-\mathrm{v}\right)-k_{0}\left(a_{0}-k_{N 1}^{\prime} u / k_{N 1} \mathrm{v}\right)-3\left(k_{N 5}+k_{0}\right)\left(k_{N 3} \mathrm{v}^{2} /\right.$ $\left.\left.k_{N 4} u\right)\right) / \Delta$,

$$
\begin{aligned}
& A_{11}=1+\frac{6 k_{N 3} \mathrm{v}}{k_{N 4} u}+\frac{k_{N 1}^{\prime} u}{k_{N 1} \mathrm{v}^{2}}, A_{12}=\frac{k_{N 1}^{\prime} u}{k_{N 1} \mathrm{v}^{2}}+\frac{2 k_{N 3} \mathrm{v}}{k_{N 4} u}, \\
& A_{22}=1+\frac{k_{N 1}^{\prime}}{k_{N 1} \mathrm{v}}+\frac{k_{N 3} \mathrm{v}^{2}}{k_{N 4} u^{2}}, \\
& A_{21}=\frac{k_{N 1}^{\prime}}{k_{N 1} \mathrm{v}}+\frac{3 k_{N 3} \mathrm{v}^{2}}{k_{N 4} u^{2}}
\end{aligned}
$$

and $\Delta=A_{11} A_{22}-A_{12} A_{21}$. Now, the whole calculation is consistent provided that $\left|\epsilon a^{(1)}\right| \ll\left|a^{(0)}\right|$ and $\left|\epsilon z^{(1)}\right| \ll\left|z^{(0)}\right|$, but both $a^{(1)}$ and $z^{(1)}$ contain terms that are proportional to $\partial u / \partial t$ and $\partial \mathrm{v} / \partial t$ (see Ref. 24). These time derivatives may get large given that Eqs. (41)-(42) contain terms which are proportional to $1 / \epsilon$. However, the existence of more than two time scales is of help in this case. Assuming that the righthand side of Eqs. (41) and (42) are dominated by the terms proportional to $1 / \epsilon$, we may rewrite the conditions $\left|\epsilon a^{(1)}\right|$ $\ll\left|a^{(0)}\right|$ and $\left|\epsilon z^{(1)}\right| \ll\left|z^{(0)}\right|$ as (see Ref. 24):

$$
\begin{aligned}
& \frac{k_{N 3}}{k_{N 1}}\left|\frac{\mathrm{v}}{u \Delta}\left(1+\frac{2 k_{N 3} \mathrm{v}}{k_{N 4} u}\right)+\frac{1}{\Delta}\right| \ll 1, \\
& \frac{k_{N 3}}{k_{N 4}}\left|\frac{2 \mathrm{v}}{\Delta}+\frac{\mathrm{v}^{2}}{u \Delta}\right| \ll 1 .
\end{aligned}
$$

For the parameter values we are considering it is $k_{N 3} / k_{N 1}$ $=1.5 \times 10^{-6}$ and $k_{N 3} / k_{N 4}=3.26 \times 10^{-5}$. Thus, provided that $\mathrm{v} / u$ does not become too large, the conditions (43) and (44) are satisfied and the calculation is self-consistent. As in the Selkov model, also in this case we get cross-diffusion terms which are small for $k_{N 3} / k_{N 4}$, and $k_{N 1}^{\prime} / k_{N 1} \vee$ small enough. Under these conditions, however, the rescaling of the diffusion coefficients is not very important and the ratio between them may be approximated by

$$
D_{u} / D_{\mathrm{v}} \approx 1+\frac{6 k_{N 3} \mathrm{v}}{k_{N 4} u}+\frac{k_{N 1}^{\prime} u}{k_{N 1} \mathrm{v}^{2}}-\frac{k_{N 1}^{\prime}}{k_{N 1} \mathrm{v}}-\frac{k_{N 3} \mathrm{v}^{2}}{k_{N 4} u^{2}} .
$$

For some values of $u$ and $\mathrm{v}$ of interest for the experimental system this ratio becomes larger than one. As we discuss in Ref. 31, the dependence of the rescaled coefficients with the concentrations must be taken into account in this case. Furthermore, via an approximate local analysis we have recently found that the reduction does not hold for all the concentration values that may be achieved in the experiment. How- 
ever, in the regions where it holds, we may conclude that the rescaling is indeed enough to explain the formation of the observed patterns. ${ }^{31}$ In any case, a more detailed analysis of the full reduced set of equations is still necessary.

\section{CONCLUSIONS}

We have used a multiple scale analysis ${ }^{23}$ to study the slow-time dynamics of reaction-diffusion systems with several time scales. The method is particularly suitable for the case of reversible reactions. We have also approached the reduction in a way similar to the quasisteady-state approximation used for ordinary differential equations. ${ }^{21,22}$ Both approaches are the same in the case of one fast reversible reaction. The reduced equations are usually a generalization of reaction-diffusion equations, since they involve crossdiffusion terms and diffusion coefficients that are concentration-dependent. Thus, these diffusion coefficients vary in space through their dependence on the concentrations. If taking them as constant is a good approximation, then the cross-diffusion terms may be eliminated by a linear transformation. In this case, the resulting (reduced) equations are of the usual reaction-diffusion type, but for variables that do not correspond to the concentration of any actual chemical species (they correspond to linear combinations of the concentrations).

In cases involving several fast reactions, some of them irreversible, it is easier to follow the steps in the quasisteadystate style. However, in this case, the validity of the reduction is not known a priori and an a posteriori check is necessary. We have analyzed in this way the Selkov model and the FIS reaction.

The Selkov model is a model for the glycolytic oscillations that describes the conversion of ATP into ADP. The possibility of developing Turing patterns within the small scales of the cell for this pathway was discussed in Ref. 14. It required that ATP and ADP diffused at unequal rates, which seemed unrealistic given the similarities in their structure. However, they both react differently with enzymes that may be assumed to remain immobile. Thus, we reduced the evolution equations involving ATP, ADP, and the enzymes to a system that described the slow dynamics of ATP and ADP. We wanted to determine if the resulting system could be of reaction-diffusion type with diffusion coefficients rescaled in the right way. We concluded that under the experimental conditions in vitro, this rescaling is very small, while for other conditions that may hold in vivo, the resulting system involves cross-diffusion terms. A further analysis of this more complicated system is necessary in order to achieve any conclusion on the possibility of developing patterns. We will do this in the future.

The interest in the FIS reaction was motivated by the observation of a variety of patterns that could be reproduced in numerical simulations of very simple models. The existence of patterns in the models also required different diffusivities. In this case again we investigated if the reduction of the original system to a set of two equations could account for this difference. However, as in the case of the Selkov model, the rescaling was not important when the reduced system was of the usual reaction-diffusion type in the original variables, with negligible cross-diffusion terms. When these cross-diffusion terms (with concentration-dependent "diffusion coefficients") become important, a further analysis is necessary. By means of a local analysis we have recently found that, under the experimental conditions of Ref. 15 , the rescaling may account for the formation of the patterns. ${ }^{31}$

\section{ACKNOWLEDGMENTS}

This work was supported by the University of Buenos Aires, CONICET (Argentina), and FOMEC. We thank J. Pearson for useful discussions.

${ }^{1}$ M. C. Cross and P. C. Hohenberg, Rev. Mod. Phys. 65, 851 (1993).

${ }^{2}$ J. D. Murray, Mathematical Biology (Springer, New York, 1989).

${ }^{3}$ A. M. Turing, Philos. Trans. R. Soc. London, Ser. B 237, 37 (1952).

${ }^{4}$ S. Kondo, and R. Asai, Nature (London) 376, 765 (1995).

${ }^{5}$ V. Castets, E. Dulos, J. Boissonade, and P. De Kepper, Phys. Rev. Lett. 64, 2953 (1990); Q. Ouyang and H. Swinney, Nature (London) 352, 610 (1991); P. DeKepper, V. Castes, E. Dulos, and J. Boissonade, Physica D 49, 161 (1991); Q. Ouyang and H. Swinney, Chaos 1, 411 (1991).

${ }^{6}$ I. Lengyel and E. Epstein, Science 251, 650 (1991).

${ }^{7}$ J. E. Pearson and W. Bruno, Chaos 2, 513 (1992).

${ }^{8}$ S. P. Dawson, A. Lawniczak, and R. Kapral, J. Chem. Phys. 100, 5211 (1994).

${ }^{9}$ J. Wagner and J. Keizer, Biophys. J. 67, 447 (1994).

${ }^{10}$ N. L. Allbritton, T. Meyer, and L. Stryer, Science 258, 1812 (1992).

${ }^{11}$ E. E. Selkov, Eur. J. Biochem. 4, 79 (1968).

${ }^{12}$ E. C. Edblom, M. Orban, and I. R. Epstein, J. Am. Chem. Soc. 108, 2826 (1986).

${ }^{13}$ A biologically relevant metabolic pathway responsible for the conversion of glucose in carbon dioxide and water in cells with production of ATP.

${ }^{14}$ B. Hasslacher, R. Kapral, and A. Lawniczak, Chaos 3, 7 (1993). Actually, the model considered in this article is an extension of the one originally introduced by Selkov. In any case, the differences between these two models do not affect our conclusions.

${ }^{15}$ K. J. Lee, W. D. McCormick, Q. Ouyang, and H. L. Swinney, Science 261, 192 (1993); K. J. Lee, W. D. McCormick, J. E. Pearson, and H. L. Swinney, Nature (London) 369, 215 (1994).

${ }^{16}$ K. J. Lee and H. L. Swinney, Phys. Rev. E 51, 1899 (1995).

${ }^{17}$ W. Reynolds, J. E. Pearson, and S. P. Dawson, Phys. Rev. Lett. 72, 2797 (1994); W. Reynolds, S. P. Dawson, and J. E. Pearson, Phys. Rev. E 56, 185 (1997); S. P. Dawson, M. V. D’Angelo, and J. E. Pearson (in preparation).

${ }^{18}$ A. Hagberg, and E. Meron, Chaos 4, 477 (1994).

${ }^{19}$ J. Guckenheimer and P. Holmes, Nonlinear Oscillations, Dynamical Systems and Bifurcations of Vector Fields (Springer, New York, 1986).

${ }^{20} \mathrm{R}$. Temam, Infinite-Dimensional Dynamical Systems in Mechanics and Physics (Springer, New York, 1997).

${ }^{21}$ C. L. Frenzen and P. K. Maini, J. Math. Biol. 26, 689 (1988).

${ }^{22}$ L. A. Segel and M. Slemrod, SIAM (Soc. Ind. Appl. Math.) Rev. 3, 466 (1989).

${ }^{23}$ E. J. Hinch, Perturbation Methods (Cambridge University Press, Mexico, 1992), p. 116.

${ }^{24}$ See EPAPS document No. E-JCPSA6-111-518948 for a detailed derivation. This document may be retrieved via the EPAPS homepage (http:// www.aip.org/pubservs/epaps.html) or from ftp.aip.org in the directory /epaps/. See the EPAPS homepage for more information.

${ }^{25}$ See, e.g., R. Kapral, S. Consta, and L. McWhirther, in Classical and Quantum Dynamics in Condensed Phase Systems, edited by B. J. Berne, G. Ciccotti and D. Coker (World Scientific, Singapore, 1998), p. 583; also at http://www.chem.utoronto.ca/ rkapral/Papers/lericif/lericif.html for a discussion about the limitations of this approach.

${ }^{26}$ In order to simplify the notation we do not write explicitly the dependence of the concentrations on $\mathbf{x}$, but actually the concentrations are functions of both $\mathbf{x}, T$ and $\tau$. 
${ }^{27}$ An enzyme is said to be allosteric when its affinity for a given molecule can be enhanced or reduced when another molecule is bound to a specific binding site of it.

${ }^{28}$ A more accurate model for the glycolytic pathway was proposed by A. Goldbeter, see e.g., Biochemical Oscillations and Cellular Rhythms. The
Molecular Bases of Periodic and Chaotic Behaviour (Cambridge University Press, Cambridge, 1996).

${ }^{29}$ V. Gáspár and K. Showalter, J. Phys. Chem. 109, 4869 (1987).

${ }^{30}$ V. Gáspar and K. Showalter, J. Phys. Chem. 94, 4973 (1990).

${ }^{31}$ M. V. D'Angelo, S. Ponce Dawson, and J. E. Pearson (in preparation). 